from capping by proteins that would block elongation ${ }^{14}$. By contrast, Arp2/3 binds at the pointed end of the growing filament, leaving the barbed end unprotected.

Finally, in terms of their role in adhesion, formins might anchor a new filament to the junction and allow the elongating filament to sustain tension. Formin at the barbed end helps to localize the growing filament, and although it is unknown how much tension can be sustained by the link between a formin and the filament, it must be more than can be sustained by an Arp2/3-nucleated filament where the barbed end is free. Indeed, several formininduced bundles, including stress fibres and radial cables of adherens junctions, are under myosin-induced tension. In the case of either formin or Arp2/3, freshly nucleated filaments would promptly be anchored by actin-binding proteins (including myosin VI (ref. 15), $\alpha$-actinin and $\operatorname{VASP}^{16}$ ), which rapidly bind and release a filament, thereby sustaining tension while still allowing filament slippage.

Thus, it seems likely that both Arp2/3 and formins contribute to junction formation. Nucleation by the Arp2/3 complex probably allows the surface protrusions from neighbouring cells to make contact and initiate the junction formation. Formins might then be enlisted to mechanically strengthen the cytoplasmic face of the junction.

1. Perez-Moreno, M. Jamora, C. \& Fuchs, E. Cell 112, 535-548 (2003).

2. Vasioukhin, V. et al. Cell 100, 209-219 (2000).

3. Kobielak, A. Pasolli, H. \& Fuchs, E. Nature Cell Biol. 6, 21-30 (2004)
4. Wallar, B. J. \& Alberts, A. S. Trends Cell Biol. 13, 435-466 (2003).

5. Li, F. \& Higgs, H. N. Curr. Biol. 13, 1335-1340 (2003).

6. Watanabe, N. et al. Nature Cell Biol. 1, 136-143 (1999).

7. Riveline, D. et al J. Cell Biol. 153, 1175-1186 (2001).

8. Afshar, K., Stuart, B. \& Wasserman, S. A. Development 127, 1887-1897 (2000).

9. Wheelock, M. J. \& Johnson, K. R. Curr. Opin. Cell Biol. 15, 509-514 (2003).

10. Sahai, E. \& Marshall, C. J. Nature Cell Biol. 4, 408-415 (2002)

11. Geiger, B. \& Bershadsky, A. Cell 110, 139-142 (2002).

12. Turnacioglu, K. K., Sanger, J. W. \& Sanger, J. M. Cell Motil. Cytokel. 40, 59-70 (1998).

13. Sechi, A. S., Wehland, J. \& Small J. V. J. Cell Biol. 137, 155-167 (1997).

14. Zigmond, S. H. et al. Curr. Biol. 13, 1820-1823 (2003).

15. Geisbrecht, E. R. \& Montell, D. J. Nature Cell Biol. 4, 616-620 (2002).

16. Laurent, V. et al. J. Cell Biol. 144, 1245-1258 (1999).

\section{The centrosome yields its secrets}

The axiom 'garbage in, garbage out' defines the limits of even the most powerful of analytical tools in molecular biology. We have the ability, for example, to determine the protein composition of practically any biological sample using mass spectrometry. But if the original sample is of low quality, the resulting reams of data can be almost meaningless. A paper by Andersen et al. (Nature 426, 570-574 (2003)) describes a new technique to extract mass spectrometry information from complex samples of only modest purity, and applies it to one of the most interesting of organelles - the centrosome.

In animal cells, the centrosome nucleates microtubule polymerization, anchors those microtubules to create arrays that are capable of work, and duplicates exactly once per cell cycle. The centrosome is unique because it is has a discrete size and structure, even though it lacks a membrane to separate it from the rest of the cytoplasm. Since the mid-1980s, approximately 60 proteins associated with the centrosome have been described. Most of these are defined as such only by immunofluorescence microscopy localization of the protein to the centrosome, and few of them have known functions. It is clear that there are many more proteins in the centrosome, but how can we identify them?

The problem with identifying centrosome proteins is that this small organelle is difficult to purify and comprises only a tiny fraction of total cell protein. Mass spectrometry has the sensitivity to overcome this problem, but there is still the problem of purity. Andersen et al. used a straightforward variation of standard mass spectrometry approaches, which they call "protein correlation profiling", allowing many contaminating proteins to be eliminated at the analysis stage. The basis for this is intuitively obvious to anyone who has purified a protein on the basis of activity: genuine centrosome proteins should co-fractionate in a purification step, whereas contaminating proteins will not usually cofractionate with the centrosome proteins. The authors analysed the relative abundance of the mass spectrometry peptides in the peak centrosome fraction and in surrounding fractions from the last step of centrosome purification; they focused on those proteins corresponding

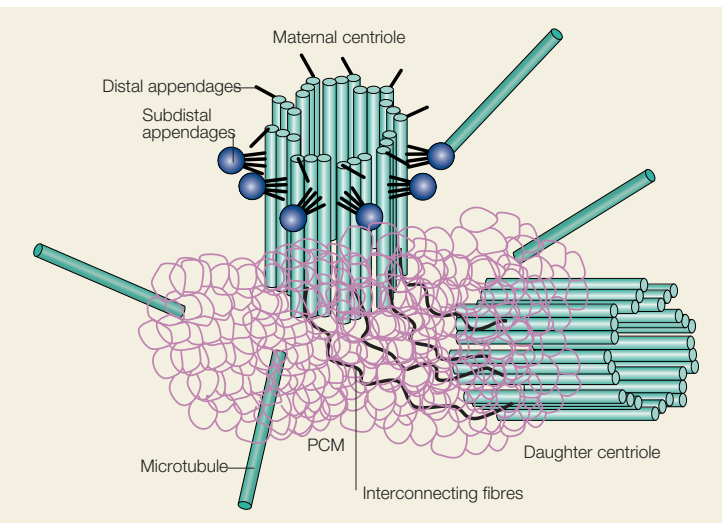

The centrosome is composed of two centrioles, connected by interconnecting fibres and surrounded by the pericentriolar material (PCM). Microtubules are nucleated from the PCM and the appendages of the maternal centriole. With the new work from Anderson et al., over 80 proteins have been localized to this complex. Figure adapted with permission from Nature Reviews Mol. Cell Biol. 2, 688-698 () (2001) Macmillan Magazines Ltd.

to peptides that co-fractionated with known centrosomal proteins.

It was reassuring to find that this approach identified most of the known centrosomal proteins. More importantly, however, Andersen et al. identified 23 new putative centrosome components and validated their analysis by examining the localization of green fluorescent protein (GFP)-tagged versions of each component. Remarkably, all 23 localized to the centrosome in transfected cells. So what does this treasure trove of new centrosome proteins reveal about centrosome structure and function? At this point rather little, except for the observation that many of the proteins have a coiled-coil structure. However, much more is sure to come. Equally exciting is the prospect of using the protein correlation profiling method to crack other difficult proteomic projects for which sample purity is limiting.

TIM STEARNS

Tim Stearns is at the Department of Biological Sciences, Stanford University, Stanford, CA 94305-5020, USA.

e-mail: stearns@stanford.edu 